\title{
A SAMPLING THEOREM FOR PERIODIC PIECEWISE POLYNOMIAL SIGNALS
}

\author{
Martin Vetterli ${ }^{1,2}$, Pina Marziliano ${ }^{1}$ and Thierry Blu ${ }^{3}$ \\ ${ }^{1} \mathrm{LCAV},{ }^{3} \mathrm{BIG}$, Ecole Polytechnique Fédérale de Lausanne, Switzerland \\ ${ }^{2}$ EECS Dept., University of California at Berkeley, USA \\ email: [martin.vetterli,pina.marziliano,thierry.blu] depfl.ch
}

\begin{abstract}
We consider the problem of sampling signals which are not bandlimited, but still have a finite number of degrees of freedom per unit of time, such as, for example, piecewise polynomials. We demonstrate that by using an adequate sampling kernel and a sampling rate greater or equal to the number of degrees of freedom per unit of time, one can uniquely reconstruct such signals. This proves a sampling theorem for a wide class of signals beyond bandlimited signals. Applications of this sampling theorem can be found in signal processing, communication systems and biological systems.
\end{abstract}

\section{INTRODUCTION}

The sampling theorem is pervasive in signal processing [3] and allows to represent the class of bandlimited signals by appropriate samples of the signal (e.g. taken at twice the maximum frequency). When signals live on a subspace spanned by a basis function and its shifts $\{\varphi(t-n T)\}_{n \in \mathbb{Z} \text {, }}$ they can be reconstructed from samples derived from inner products as well. But in general, signals which do not live on a specific subspace can only be reconstructed up to the projection onto that subspace (e.g. the bandlimited approximation).

In this paper, we consider classes of signals which are not bandlimited nor live on subspaces, yet can be represented through sampling. The key property of these signals is that they have a finite number of degrees of freedom per unit of time, what we call a finite rate of innovation [4].

Examples of such signals are bilevel signals with a finite number of transitions per unit of time. Since the signal is discontinuous, it is clearly not bandlimited, yet, because of the finite rate of transition, it is possible to derive a sampling scheme from which the signal can be perfectly reconstructed.

Instead of sampling the signal $x(t)$ directly, we sample the output of $x(t)$ convolved with a kernel $\tilde{\varphi}(t)=\varphi(-t)$, so that the samples taken at integer multiples of $T$ are

$$
x[n]=<x(t), \varphi(t-n T)>.
$$

This model corresponds to the usual physical set up where the signal is "seen" through a channel, or passed through a lowpass filter before sampling.

The key question we pursue in this paper is thus: for what signals with finite rate of innovation and what sampling kernels can we perfectly recover $x(t)$ from regular sampling? After a definition of signals with finite rate of innovation, we demonstrate a sampling theorem for streams of Diracs and periodic piecewise polynomial signals. We also show local reconstruction algorithms based on splines for some simple signals.

\section{DEFINITIONS}

Let us start by defining the classes of real signals we are considering in the sequel.

Definition 1 A signal with finite rate of innovation is a function of time which allows a parametric representation having a finite number of degrees of freedom over finite windows of time.

Definition 2 The rate of innovation $\rho$ is the average number of degrees of freedom per unit of time, or, with $C_{x}\left(t_{0}, t_{1}\right)$ giving the number of degrees of freedom of $x(t)$ over the interval $\left[t_{0}, t_{1}\right]$,

$$
\rho=\lim _{T \rightarrow \infty} \frac{1}{T} C_{x}\left(-\frac{T}{2}, \frac{T}{2}\right) .
$$

If we consider finite length or periodic signals of length $T$, then the number of degrees of freedom is finite, and the rate of innovation is $1 / T C_{x}[0, T]$. Bandlimited signals with support $[-\pi / T, \pi / T]$ have a rate of innovation of $1 / T$, since they are uniquely specified by samples taken every $T$ seconds. If we consider discrete-time sequences then general sequences have a (normalized ) rate of innovation of 1 (one degree of freedom per sample).

Example 1 Poisson process.

A Poisson process generates Diracs with independent and identically distributed (i.i.d.) interarrival times, the distribution being exponential with probability density function 
$\mu e^{-\mu t}$. The expected interarrival time is given by $1 / \mu$. Thus, the rate of innovation is $\mu$.

While one can define many parametric signals which have a finite rate of innovation, in the sequel we will concentrate on streams of Diracs and piecewise polynomials which are classes for which we are able to give sampling theorems and reconstruction formulae.

\section{DISCRETE-TIME SIGNALS WITH FINITE RATE OF INNOVATION}

We will start with the simplest case, namely discrete-time periodic signals. Among them, stream of Diracs are the most elementary and thus we start with them. More generally, piecewise polynomials can be reduced to stream of Diracs through appropriate derivation, which is done next.

\subsection{Stream of Diracs}

Consider a discrete-time periodic signal

$$
\mathbf{x}=(x[0], x[1], \ldots, x[N-1])^{T}
$$

containing $K$ weighted Diracs at locations $\left\{n_{k}\right\}_{k=0}^{K-1}$, or

$$
x[n]=\sum_{k=0}^{K-1} c_{k} \delta\left[n-n_{k}\right],
$$

where $\delta[n]$ is the Kronecker $\delta[n]=1$ if $n=0,0$ else. Call $\mathbf{X}$ the discrete-time Fourier series (DTFS) coefficients of $\mathbf{x}$ where

$$
X[m]=\sum_{k=0}^{K-1} c_{k} W_{N}^{n_{k} m}, \quad m=0, \ldots, N-1
$$

and $W_{N}=e^{-i 2 \pi / N}$. $\mathbf{X}$ is thus a linear combination of complex exponentials, each of which can be cancelled with an appropriate zero at location $W_{N}^{n_{k}}$. Given $\mathbf{X}$, it suffices to find the annihilating filter ${ }^{1} \mathbf{H}=(1, H[1], \ldots, H[K])$ satisfying [2]

$$
\mathbf{H} *_{c} \mathbf{X}=0 .
$$

This filter $\mathbf{H}$ has $z$-transform $H(z)$ which factors as

$$
H(z)=\prod_{k=0}^{K-1}\left(1-z^{-1} W_{N}^{n_{k}}\right)
$$

having zeros at $W_{N}^{n_{k}}, k=0, \ldots, K-1$. Hence to find the set of locations $\left\{n_{k}\right\}_{k=0}^{K-1}$ it suffices to find the filter coefficients $\{H[k]\}_{k=1}^{K}$ and then find the zeros of $H(z)$. Equation (6) leads to a Toeplitz system of equations involving

\footnotetext{
${ }^{1}$ This is also known as the error locator polynomial in error correction coding.
}

$2 K$ successive components of $\mathbf{X}$ and this system is always solvable. Typically, one uses the $2 K$ central terms of $\mathbf{X}$, which correspond to an ideal lowpass version of $\mathbf{x}$. In that case, $x[n]$ can be convolved with an ideal sinc filter that keeps frequencies between $-K$ and $K$. The result of the convolution can be subsampled by an integer $M$, as long as $M$ is a divisor of $N$ and $M$ is small enough such as to avoid aliasing.

Finally, to find the $K$ values $\left\{c_{k}\right\}_{k=0}^{K-1}$ we need to solve the Vandermonde system in (5) with $m=0, \ldots, K-1$, which is also always solvable. Putting all together, we have

Proposition 1 Consider a discrete-time periodic signal $x[n]$ of period $N$ containing $K$ weighted Diracs. Let $M$ be an integer divisor of $N$ satisfying $N / M \geq 2 K+1$ and take the discrete-time periodized sinc sampling kernel $\varphi[n]=$ $\frac{1}{N} \sum_{m=-K}^{K} W_{N}^{-m n}$, that is, the inverse DTFS of Rect ${ }_{[-K, K]}$. Then the $N / M$ samples

$$
y_{s}[l]=\langle x[n], \varphi[n-l M]\rangle \quad l=0, \ldots, N / M-1(8)
$$

are a sufficient representation of the signal.

Expanding (8), we get $y_{s}[l]=\frac{1}{N} \sum_{m=-K}^{K} X[-m] W_{N / M}^{m l}$ from which are obtained $N / M$ values $X[m]$ sufficient to find the locations of the Diracs. See [5] for a detailed proof. When $N / M=2 K+1$ then the number of samples in (8) is just 1 more than the number of degrees of freedom ${ }^{2}$, that is, we are very close to "critical sampling".

\subsection{Piecewise Polynomial Signals}

Define a discrete-time piecewise polynomial signal by $K$ intervals $\left[n_{k}, n_{k+1}\right]$ and pieces which are polynomials of maximum degree $R$ on $\left[n_{k}, n_{k+1}-1\right]$. Note that this is more general than integrating a stream of $K$ Diracs (4), since for example discontinuities at interval boundaries are permitted. To extend the above result to piecewise polynomials of maximum degree $R$, we need to take $(R+1)$ first order discrete-time differences, and then apply Proposition 1. Call $d[n]=\delta[n]-\delta[n-1]$ the discrete-time periodic first order difference, with $D[m]=1-W_{N}^{m}$ its DTFS coefficients. Call $\varphi[n]$ the discrete-time periodized sinc kernel of appropriate bandwidth. Then the $(R+1)$ th derivative sinc sampling kernel $\psi[n]$ is given by $\psi[n]=$ $\underbrace{d[n] * d[n] * \cdots * d[n]}_{R+1} * \varphi[n]$. The convolution of the signal $x[n]$ with $\psi[n]$ is equivalent (by associativity of the convolution operator) to convolving the $(R+1)$ th (discretetime) derivative of $x[n]$ with the sinc kernel $\varphi[n]$. Now, this

\footnotetext{
${ }^{2}$ In the discrete-time scenario, position is not a "real" degree of freedom, since it is an integer. Thus, combinatorial methods can actually work with fewer "samples" in certain cases [1]
} 


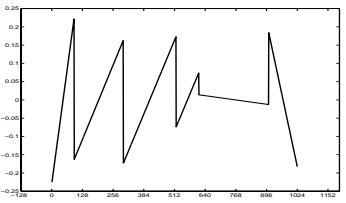

(a)

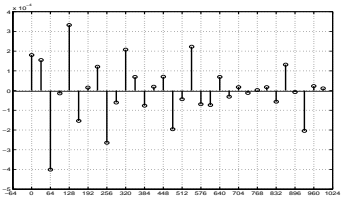

(c)

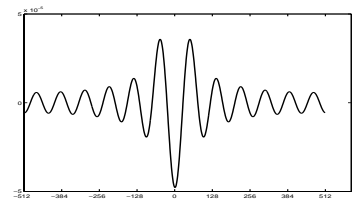

(b)

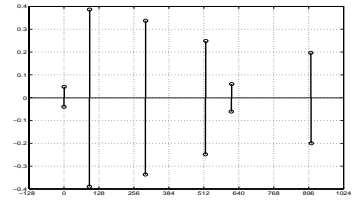

(d)
Figure 1: (a) Piecewise linear $(R=1)$ signal of period $N=$ 1024 with $K=6$ pieces; (b) Differentiated sinc sampling kernel, $\psi[n]=d[n] * d[n] * \varphi[n]$; (c) Sample values $y_{s}[l]=<$ $x[n], \psi[n-l M]>$ with $M=32$; (d) Stream of $K(R+1)=$ 12 weighted Diracs, $x^{(2)}[n]=d[n] * d[n] * x[n]$.

$(R+1)$ th derivative, $x^{(R+1)}[n]$ is a collection of at most $K(R+1)$ weighted Dirac spikes from which $x[n]$ can be recovered (up to polynomials of degree smaller than $(R+1)$ ). Since we know how to reconstruct $x^{(R+1)}[n]$ from the inner products with the sinc kernel, we have

Theorem 1 Consider a zero mean discrete-time periodic piecewise polynomial signal of period $N$ with $K$ pieces of degree $R$. Let $M$ be an integer and a divisor of $N$ such that $N / M \geq 2 K(R+1)+1$. Take a sampling kernel $\psi[n]$ with DTFS $\Psi[m]=(D[m])^{R+1} \operatorname{Rect}_{[-K(R+1), K(R+1)]}$. Then we can recover the signal from the $N / M \in \mathbb{N}$ samples

$$
y_{s}[l]=<x[n], \psi[n-l M]>\quad l=0, \ldots, N / M-1 .
$$

For a proof see [5]. Figure 1 illustrates the reconstruction of a discrete-time periodic piecewise linear $(R=1)$ signal of period $N=1024$ with $K=6$ pieces. We take $N / M=32$ samples. The reconstructed signal is equal to the original (Fig. 1(a)) within machine precision, $10^{-11}$.

\section{CONTINUOUS-TIME SIGNALS WITH FINITE RATE OF INNOVATION}

We derive now the equivalent results but in continuous time, again building up from stream of Diracs to piecewise polynomial signals.

\subsection{Stream of Diracs}

Consider a periodic signal $x(t)$ of period $\tau$ containing $K$ Diracs at locations $\left\{t_{k}\right\}_{k=0}^{K-1}$ with $t_{k} \in[0, \tau)$, or

$$
x(t)=\sum_{n \in \mathbb{N}} \sum_{k=0}^{K-1} c_{k} \delta\left(t-n \tau-t_{k}\right) .
$$

Consider the continuous-time Fourier series (CTFS) coefficients of $x(t)$

$$
X[m]=\frac{1}{\tau} \int_{0}^{\tau} x(t) e^{-i 2 \pi m t / \tau} d t=\frac{1}{\tau} \sum_{k=0}^{K-1} c_{k} e^{-i 2 \pi m t_{k} / \tau}
$$

Assume we convolve $x(t)$ with a periodic sinc filter of bandwidth $[-K, K]$. This leads to a lowpass approximation $y(t)$ given by

$$
y(t)=\sum_{m=-K}^{K} X[m] e^{i 2 \pi m t / \tau} .
$$

Consider now sampling $y(t)$ at multiples of $T$, where $\tau / T \in$ $\mathbb{N}$

$$
y_{s}[l]=y(l T)=\sum_{m=-K}^{K} X[m] e^{i 2 \pi m l T / \tau} .
$$

Clearly, as long as $\tau / T \geq 2 K+1$, (13) can be used to recover $X[m]$. To find the $t_{k}$ 's in (11), we need to find the annihilating filter $\mathbf{H}=(1, H[1], H[2], \ldots, H[K])$ such that

$$
\mathbf{H} *_{c} \mathbf{X}=0 .
$$

Consider the $z$-transform of $\mathbf{H}$, or $H(z)=\sum_{l=0}^{K} H[l] z^{-l}$ which factorizes into

$$
H(z)=\prod_{k=0}^{K-1}\left(1-z^{-1} Z_{k}\right)
$$

and we find

$$
Z_{k}=e^{-i 2 \pi t_{k} / \tau},
$$

that is, the $K$ locations $\left\{t_{k}\right\}_{k=0}^{K-1}$. The system to solve in (14) is the same Toeplitz system that we considered in the previous section. Similarly, a Vandermonde system then gives the values $\left\{c_{k}\right\}_{k=0}^{K-1}$. Therefore we can state:

Proposition 2 Consider a continuous-time periodic stream of $K$ weighted Diracs with period $\tau$ and a periodic sinc sampling kernel of bandwidth $[-K, K]$. The stream of Diracs is uniquely defined by taking $\tau / T \in \mathbb{N}$ samples $y_{s}[l]$ defined in (13), with $\tau / T \geq 2 K+1$.

The proof is found in [5].

\subsection{Piecewise Polynomial Signals}

Without getting into details at this point, we simply mimic the approach shown in the discrete-time case. The CTFS of the sampling kernel needs to have a factorization

$$
\Psi[m]=(D[m])^{R+1} \Phi[m]
$$


where $D[m]=i 2 \pi m$ is the CTFS of the derivative and $\Phi[m]$ is the appropriate Rect function. Thus, $\Psi[m]$ is the CTFS of a bandlimited $(R+1)$ th derivative. The simplest form of the result appears for piecewise polynomials of degree $R$ which have $(R-1)$ continuous derivatives (e.g. piecewise linear and continuous). The first factor in (17) leads to the $(R+1)$ th derivative of $x(t)$, which can be recovered from sampling as shown in the previous subsection, and thus [5]

Theorem 2 Consider a continuous-time periodic piecewise polynomial signal, $x(t)$, with period $\tau$ and $K$ pieces of maximum degree $R$, belonging to $\mathcal{C}^{R-1}$ and having zero mean. Consider a sampling kernel as in (17) with a Rect of width $[-K, K]$. If $\tau / T \geq 2 K+1$ then $x(t)$ can be uniquely recovered from the $\tau / T \in \mathbb{N}$ samples

$$
y_{s}[l]=<x(t), \psi(t-l T)>\quad l=0, \ldots, \tau / T-1 .
$$

\subsection{Finite length signals}

It is possible to consider using infinitely supported sampling kernels (like the sinc or the Gaussian kernel) to sample finite length signals which are piecewise polynomial. Then, a finite number of samples allows to reconstruct the signal. The techniques, while similar in spirit, are more complex, and we refer to [5] for details.

\section{LOCAL RECONSTRUCTION ALGORITHMS}

The methods seen so far require global information to reconstruct the signal, or the signal has to be of finite length. A question of interest is to see under which conditions a local reconstruction scheme is possible. To explore this, we consider the simplest piecewise polynomial, namely the bilevel signal. We then use $\beta$-splines of varying degrees as local sampling kernels.

\subsection{Bilevel Signals}

Define a bilevel signal as a continuous-time signal $x(t)=$ 0 or $1, t \in \mathbb{R}^{+}$, with $x(0)=1$. Consider first the box spline $\varphi_{0}(t)=1$ if $0 \leq t<1,0$ otherwise. Suppose $x(n)=1$ and there is a transition $t_{k}$ in $[n, n+1]$. Then the sample value $y_{s}[n]=<x(t), \varphi_{0}(t-n)>=t_{k}-n$ which implies that the transition $t_{k}=y_{s}[n]+n$. This leads us to the following proposition.

Proposition 3 A bilevel signal $x(t)$ is uniquely determined from the samples $y_{s}[n]=<x(t), \varphi_{0}(t-n)$ if and only if there is at most one transition $t_{k}$ in each interval $[n, n+1]$.

Sufficiency was shown above and necessity can be shown by counterexample, see [5].
An obvious question is: What happens when going to sampling kernels with larger support? Consider the linear spline or the hat function $\varphi_{1}(t)=1-|t|$ if $|t|<1,0$ otherwise .

Proposition 4 A bilevel signal $x(t)$ is uniquely determined from the samples $x[n]$ obtained using the hat sampling kernel $\varphi_{1}(t)$ with $T=1$ in (1) if and only if there are at most two transitions $t_{i} \neq t_{j}$ in each interval $[n, n+2]$.

When going to higher order splines, necessity carries over. Sufficiency, on the other hand requires to solve higher order polynomial equations, which becomes difficult. The details of the proofs are found in [5].

\subsection{Piecewise Polynomial Signals}

Here we just mention that when sampling piecewise polynomial signals using the box sampling kernel not only are the transition values unknown but the polynomial coefficients as well and so we need to increase the sampling rate by $R+2$ where $R$ is the degree of the polynomial. An iterative algorithm for piecewise constant and piecewise linear signals is given in [5].

\section{CONCLUSION}

In this paper, we have shown that piecewise polynomials with a rate of innovation of $\rho$ can essentially be sampled with an appropriate sampling kernel (e.g. sinc kernel) with a sampling period $T \leq 1 / \rho$ and can be perfectly recovered from these samples. This extends the idea of uniform sampling to a wider class than the classical, bandlimited signals. For example, piecewise bandlimited signals are treated in [6].

\section{REFERENCES}

[1] M. Gastpar and Y. Bresler, "On the necessary density for spectrum-blind nonuniform sampling subject to quantization", Proc. IEEE ICASSP, Istanbul, June 2000.

[2] P. Stoica and R. Moses, Introduction to Spectral Analysis, Prentice Hall, 2000.

[3] M. Unser, "Sampling-50 Years After Shannon," Proceedings of the IEEE, vol. 88, no. 4, pp. 569-587, April 2000.

[4] M. Vetterli, "Sampling of piecewise polynomial signals", LCAV Technical Report, EPFL, Switzerland, Dec. 1999.

[5] M. Vetterli and P. Marziliano and T. Blu, "Sampling signals with finite rate of innovation", submitted to IEEE Trans. Signal Processing, March 2001.

[6] M. Vetterli and P. Marziliano and T. Blu, "Sampling piecewise bandlimited signals", In Proc. Sampling Theory and Applications Workshop, Orlando, USA, May 2001. 